\title{
GENERALIZED $\gamma$-GENERATING MATRICES AND NEHARI-TAKAGI PROBLEM
}

\author{
VOLODYMYR DERKACH AND OLENA SUKHORUKOVA
}

Abstract. Let $\Gamma(f)$ be the block Hankel matrix of negative Fourier coefficients of a matrix valued function (mvf) $f \in L_{\infty}^{p \times q}(\mathbb{T})$ defined on the unit circle $\mathbb{T}$. In the present paper a matrix Nehari-Takagi problem is considered: Given a Hankel matrix $\Gamma$ and $\kappa \in \mathbb{N} \cup\{0\}$ find a mvf $f \in L_{\infty}^{p \times q}(\mathbb{T})$, such that $\|f\|_{\infty} \leqslant 1$ and $\operatorname{rank}(\Gamma(f)-\Gamma) \leqslant \kappa$. Under certain mild assumption, we establish a one-to-one correspondence between solutions of the Nehari-Takagi problem and solutions of some Takagi-Sarason interpolation problem. The resolvent matrix of the NehariTakagi problem is shown to belong to the class of so-called generalized $\gamma$-generating matrices, which is introduced and studied in the paper.

Mathematics subject classification (2010): Primary 47A56; Secondary 30E05, 47A57.

Keywords and phrases: Nehari-Takagi problem, $\gamma$-generating matrix, Hankel operator, generalized Schur class, Krě̆n-Langer factorization, linear fractional transformation.

\section{REFERENCES}

[1] V. M. Adamyan, D. Z. Arov, M. G. KreǏn, Infinite Hankel matrices and generalized problems of Caratheodory-Fejer and F. Riesz (Russian), Funktsional. Anal. i Prilozhen., 2 (1968), 1-18.

[2] V. M. Adamyan, D. Z. Arov, M. G. KreǏn, Analytic properties of the Schmidt pairs of a Hankel operator and the generalized Schur-Takagi problem, Matem. Sb. 86 (1971), 34-75.

[3] V. M. AdAmJAN, Nondegenerate unitary couplings of semiunitary operators, (Russian), Funktsional. Anal. i Prilozhen. 7 (1973), no. 4, 1-16.

[4] D. AlPay, H. Dym, On applications of reproducing kernel spaces to the Schur algorithm and rational $J$ unitary factorization. I. Schur methods in operator theory and signal processing, 89-159, Oper. Theory Adv. Appl., 18, Birkhäuser, Basel, 1986.

[5] D. Z. AROV, Regular and singular J-inner matrix functions and corresponding extrapolation problems, (Russian), Funktsional. Anal. i Prilozhen. 22 (1988), no. 1, 57-59; translation in Funct. Anal. Appl. 22 (1988), no. 1, 46-48.

[6] D. Z. AROV, $\gamma$-generating matrices, j-inner matrix-functions and related extrapolation problems, Teor. Funktsii Funktsional. Anal. i Prilozhen, I, 51 (1989), 61-67; II, 52 (1989), 103-109; translation in J. Soviet Math. I, 52 (1990), 3487-3491; III, 52 (1990), 3421-3425.

[7] D. Z. AROV AND H. DYM, J-inner matrix function, interpolation and inverse problems for canonical system, I: foundation, Integral Equations Operator Theory, 28 (1997), 1-16.

[8] D. Z. Arov And H. Dym, J-Contractive Matrix Valued Functions and Related Topics, Cambridge University Press, Cambridge, 2008.

[9] T. YA. AzIZOV AND I. S. IoKHVIDOv, Foundations of the theory of linear operators in spaces with an indefinite metric, Nauka, Moscow, 1986 (English translation: Wiley, New York, 1989).

[10] J. A. Ball, I. Gohberg AND L. Rodman, Interpolation of rational matrix functions, Operator Theory: Advances and Applications, 45, Birkhäuser Verlag, Basel, 1990, xiii+605 pp.

[11] J. A. Ball And J. W. Helton, A Beurling-Lax theorem for the Lie group $U(m, n)$ which contains most classical interpolation theory, J. Operator Theory, 9 (1983), no. 1, 107-142.

[12] V. DerKach, On Schur-Nevanlinna-Pick indefinite interpolation problem, Ukrainian Math. Zh., 55 (2003), no. 10, 1567-1587. 
[13] V. DerKach, H. Dym, On linear fractional transformations associated with generalized J-inner matrix functions, Integ. Eq. Oper. Th., 65 (2009), 1-50.

[14] V. DeRKACh AND H. DYM, Bitangential interpolation in generalized Schur classes, Complex Analysis and Operator Theory, 4 (2010) 4, 701-765.

[15] V. DerkaCh, H. Dym, A Generalized Schur-Takagi Interpolation Problem, Integ. Eq. Oper. Th., 80 (2014), 165-227.

[16] B. FRANCIS, A Course in $H_{\infty}$ Control Theory, Lecture Notes in Control and Information Sciences, 88. Springer-Verlag, Berlin, 1987.

[17] V. E. Katsnelson, A. YA. Kheifets And P. M. Yuditskit, The abstract interpolation problem and extension theory of isometric operators, in: Operators in Spaces of Functions and Problems in Function Theory, Kiev, Naukova Dumka, 1987, 83-96 (Russian).

[18] A. YA. KhEIFETS, Generalized bitangential Schur-Nevanlinna-Pick problem and the related Parseval equality, in: Teorija Funktsii Funktsional Anal i Prilozhen, (Russian) 54 (1990), 89-96.

[19] A. YA. KHEIFETS, Parametrization of solutions of the Nehari problem and nonorthogonal dynamics, Operator theory and interpolation (Bloomington, IN, 1996), 213-233, Oper. Theory Adv. Appl., 115, Birkhäuser, Basel, 2000.

[20] M. G. KREĬN AND H. LANGER, Über die verallgemeinerten Resolventen und die characteristische Function eines isometrischen Operators im Raume $\Pi_{\kappa}$, Hilbert space Operators and Operator Algebras (Proc.Intern.Conf.,Tihany, 1970); Colloq. Math. Soc. Janos Bolyai, 5, North-Holland, Amsterdam, 353-399, 1972.

[21] F. R. Gantmacher, The theory of matrices, AMS Chelsea Publishing, Providence, RI, 1998.

[22] Z. NehARI, On bounded bilinear forms, Ann. Math, 65 (1957), no. 1, 153-162. no. 2, 153-162.

[23] E. V. Neiman, An analogue of Rouché's theorem in the generalized Smirnov class, (Russian) Proceedings of the Institute of Applied Mathematics and Mechanics, 17 (2008) 148-153.

[24] V. PELLER, Hankel operators and their applications, Springer Monographs in Mathematics. SpringerVerlag, New York, 2003.

[25] L. A. PAGE, Bounded and compact vectorial Hankel operators, Trans. Amer. Math. Soc. 150 (1970), $529-539$. 